\title{
Prenylterphenyllin and Its Dehydroxyl Analogs, New Cytotoxic Substances from a Marine-derived Fungus Aspergillus candidus IF10
}

\author{
Hong Wei, Hirohiko Inada, Asami Hayashi, Kenichi Higashimoto, \\ Patamaporn Pruksakorn, Sayo Kamada, Masayoshi Arai, Shunsuke Ishida, \\ Motomasa Kobayashi
}

Received: July 19, 2007 / Accepted: August 20, 2007

(C) Japan Antibiotics Research Association

\begin{abstract}
Three novel cytotoxic substances named prenylterphenyllin (1), 4"-deoxyprenylterphenyllin (2), and 4 "-deoxyisoterprenin (3) were isolated from a cultured marine-derived fungus of Aspergillus candidus IF10 together with 4 "-deoxyterprenin (4). Their chemical structures were elucidated on the basis of 2D NMR analysis. These compounds $\mathbf{1} \sim \mathbf{4}$ showed cytotoxic activity against human epidermoid carcinoma KB cells (KB3-1) with $\mathrm{IC}_{50}$ of $8.5,3.0,2.5$, and $4.5 \mu \mathrm{g} / \mathrm{ml}$, respectively.
\end{abstract}

Keywords prenylterphenyllin, cytotoxic, marine-derived fungus, Aspergillus candidus

In recent years, marine microorganisms have been paid much attention as a significant source for new drug leads $[1,2]$. During course of our search for anticancer agent from marine-derived microbe, three novel cytotoxic substances named prenylterphenyllin (1), 4"deoxyprenylterphenyllin (2), and 4"-deoxyisoterprenin (3) were isolated from a cultured marine-derived fungus of Aspergillus candidus IF10 together with 4"-deoxyterprenin (4) (Fig. 1). In this paper, the fermentation, isolation, and structure elucidation of these compounds are presented.

The fungal strain IF10 was isolated from the marine

M. Kobayashi (Corresponding author), H. Wei, H. Inada, A. Hayashi, K. Higashimoto, P. Pruksakorn, S. Kamada, M. Arai, S. Ishida: Graduate School of Pharmaceutical Sciences, Osaka University, Yamada-oka 1-6, Suita, Osaka 565-0871, Japan, E-mail: kobayasi@phs.osaka-u.ac.jp sediment collected from a depth of $50 \mathrm{~m}$ off Gokasyo Gulf, Mie Prefecture, Japan, and identified as A. candidus by morphological analysis [3] (Fig. 2) and BLAST search of 28S rDNA sequence using DNA Data Bank of Japan (DDBJ). 28S rDNA sequence of IF10 strain (575 nucleotides) showed $100 \%$ of homology with those of

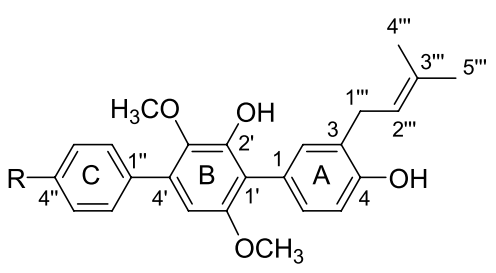

$$
\text { Prenylterphenyllin (1): } \quad \mathrm{R}=\mathrm{OH}
$$
4"-Deoxyprenylterphenyllin (2): $\mathrm{R}=\mathrm{H}$

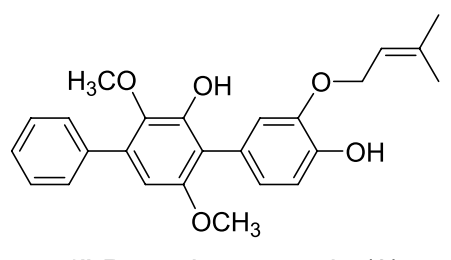

$$
\text { 4"-Deoxyisoterprenin (3) }
$$

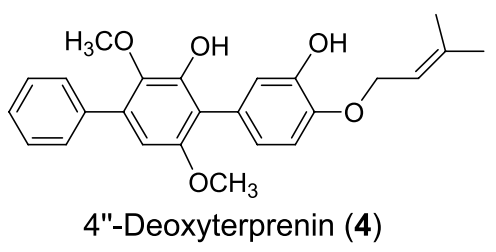

Fig. 1 Structures of prenylterphenyllins and terprenins. 
A. candidus NRRL303 (Accession No. AF433067) and A. candidus NRRL4809 (Accession No. U28765). The cytotoxic activity of these compounds was evaluated by MTT assay method using human epidermoid carcinoma $\mathrm{KB}$ cells (KB3-1). The concentration $\left(\mathrm{IC}_{50}\right)$ of test compound causing $50 \%$ inhibition of the growth of KB3-1 cells was defined as an index of cytotoxicity.

The slant culture of the strain A. candidus IF10 was inoculated into a 500-ml Erlenmeyer flask containing $200 \mathrm{ml}$ of MG medium [consisting of $2.0 \%$ malt extract (Difco, NJ, USA), $2.0 \%$ glucose, $0.1 \%$ bact peptone (Difco, NJ, USA) in artificial seawater (Yashima Pure Chemicals, Osaka, Japan)] at $30^{\circ} \mathrm{C}$ for 3 days. Then, $40 \mathrm{ml}$ aliquots of the culture were transferred into 5-liter Erlenmeyer flasks containing 1.0 liter of MG medium and cultured under static conditions at $30^{\circ} \mathrm{C}$ for 2 weeks. A typical time course of the fermentation is shown in Fig. 3. 1 was detected in the culture broth at day 10 after inoculation, and its concentration reached level of $4.5 \mathrm{mg} /$ liter at day 14 . The 2

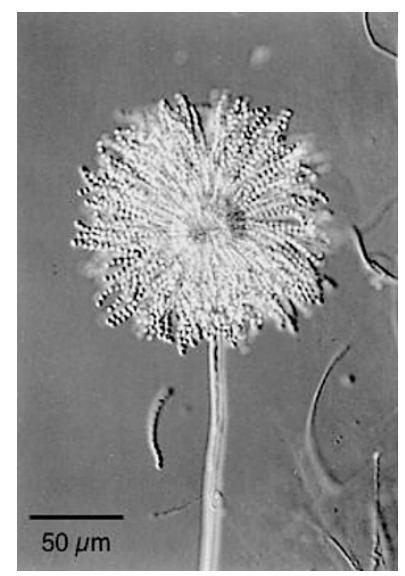

Fig. 2 Aspergillus candidus IF10 under microscope. weeks old culture was extracted with 2-butanone, and the 2-butanone-soluble portion was further partitioned into an $n$-hexane-90\% aq $\mathrm{MeOH}$ mixture to furnish a $\mathrm{MeOH}$ extract $(3.8 \mathrm{~g})$. The $\mathrm{MeOH}$ extract $(2.1 \mathrm{~g})$ was fractionated by $\mathrm{SiO}_{2}$ gel column chromatography ( $n$-hexane -EtOAc) to give eight fractions $(\mathrm{A} \sim \mathrm{H})$ on the guidance of bioassay. Fraction $\mathrm{C}(73 \mathrm{mg})$ was then subjected to $\mathrm{SiO}_{2}$ gel column chromatography ( $n$-hexane $\left.-\mathrm{CHCl}_{3}-\mathrm{EtOAc}=4: 1: 1\right)$ to afford 1 (14 mg). Fraction A (50 mg) was separated by $\mathrm{SiO}_{2}$ gel column chromatography ( $n$-hexane- $\mathrm{CHCl}_{3}$ $\mathrm{EtOAc}=10: 10: 1)$ to give four fractions (A-1 to A-4). Fraction A-4 (14 mg) was purified by HPLC (Cosmosil 5SL-II, $n$-hexane $\left.-\mathrm{CHCl}_{3}-\mathrm{EtOAc}=4: 4: 1\right)$ to furnish 2 $(2.5 \mathrm{mg})$. Then, fraction A-3 $(20 \mathrm{mg})$ was separated by HPLC (Cosmosil 5SL-II, $n$-hexane- $\mathrm{CHCl}_{3}-\mathrm{EtOAc}=$ $5: 5: 1)$ to obtain an active fraction A-3-1 $(8.0 \mathrm{mg})$, which was further purified by reversed phase HPLC (Cosmosil $5 \mathrm{C}_{18}$-MS-II, $\left.\mathrm{CH}_{3} \mathrm{CN}-\mathrm{H}_{2} \mathrm{O}=55: 45\right)$ to afford $3(1.0 \mathrm{mg})$ and 4 (4.5 mg).

The ESI TOF-MS of 1 [White powder. IR $v_{\max }(\mathrm{KBr})$ $\mathrm{cm}^{-1}: 3420,2936,1610,1522$. UV $\lambda_{\max }^{\mathrm{MeOH}} \mathrm{nm}(\varepsilon): 205$ (40200), 277 (20000). ESI TOF-MS: $m / z 429(\mathrm{M}+\mathrm{Na})^{+}$. HR-ESI TOF-MS: found $m / z 429.1660(\mathrm{M}+\mathrm{Na})^{+}$. Calcd for $\mathrm{C}_{25} \mathrm{H}_{26} \mathrm{O}_{5} \mathrm{Na}$ : 429.1678.] showed a quasi-molecular ion peak at $m / z 429(\mathrm{M}+\mathrm{Na})^{+}$, and the molecular formula was determined as $\mathrm{C}_{25} \mathrm{H}_{26} \mathrm{O}_{5}$ by HR-ESI TOF-MS in conjunction with NMR analysis. The ${ }^{1} \mathrm{H}$ - and ${ }^{13} \mathrm{C}-\mathrm{NMR}$ analyses indicated that 1 possesses an isoprenyl group $\left[\delta_{\mathrm{H}}\right.$ $5.39(1 \mathrm{H}, \mathrm{m}) ; 3.39(2 \mathrm{H}, \mathrm{d}, J=7.1 \mathrm{~Hz}) ; 1.78(3 \mathrm{H}, \mathrm{s}) ; 1.76$ $\left.(3 \mathrm{H}, \mathrm{s}) ; \delta_{\mathrm{C}} 134.6,121.8,29.8,25.8,17.9\right]$, two methoxyl groups $\left[\delta_{\mathrm{H}} 3.74(3 \mathrm{H}, \mathrm{s}) ; 3.45(3 \mathrm{H}, \mathrm{s}) ; \delta_{\mathrm{C}} 60.5,56.0\right]$, three phenolic hydroxyl groups $\left[\delta_{\mathrm{H}} 5.90(\mathrm{~s}) ; 5.32(\mathrm{~s}) ; 5.29(\mathrm{~s})\right]$, and eight aromatic protons $\left[\delta_{\mathrm{H}} 7.52(2 \mathrm{H}, \mathrm{d}, J=8.2 \mathrm{~Hz})\right.$; $7.21(2 \mathrm{H}, \mathrm{m}) ; 6.91(2 \mathrm{H}, \mathrm{d}, J=8.2 \mathrm{~Hz}) ; 6.88(1 \mathrm{H}, \mathrm{d}$, $J=8.9 \mathrm{~Hz}) ; 6.45(1 \mathrm{H}, \mathrm{s})]$. The detailed analysis of the

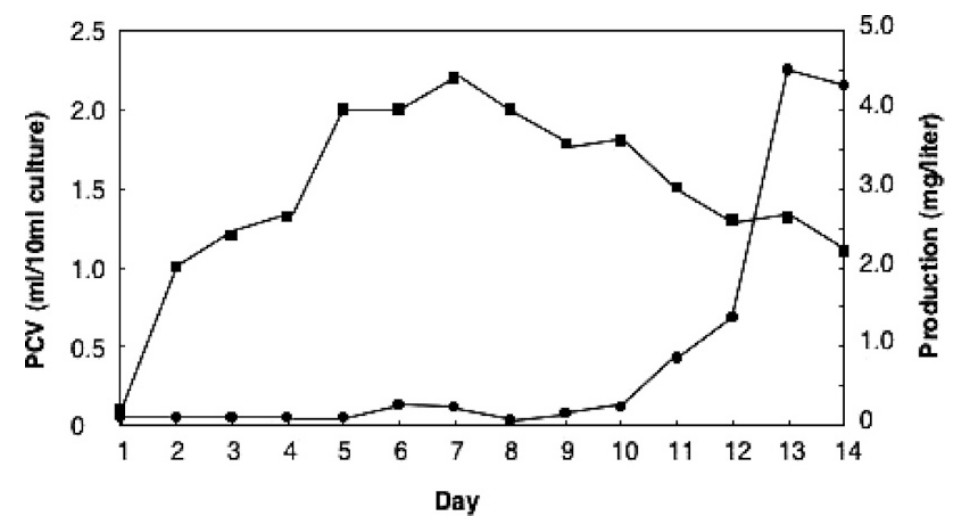

Fig. 3 Time course of prenylterphenyllin (1) production from Aspergillus candidus IF10. 
COSY, HMQC, and HMBC spectra of $\mathbf{1}$ clarified the presence of three phenyl rings and an isopentenyl moiety as shown in Fig. 4. The connection of the ring structures was also defined on the basis of the NOESY correlations of $\mathbf{1}$ (Fig. 5). Thus, the strong NOE correlations [from $\mathrm{H}-2^{\prime \prime}$ and H-6" $\left(\delta_{\mathrm{H}} 7.52,2 \mathrm{H}, \mathrm{d}, J=8.2 \mathrm{~Hz}\right)$ to $\mathrm{H}-5^{\prime}\left(\delta_{\mathrm{H}} 6.45, \mathrm{~s}\right)$ and $3^{\prime}-\mathrm{OCH}_{3}\left(\delta_{\mathrm{H}} 3.45, \mathrm{~s}\right)$; from $6^{\prime}-\mathrm{OCH}_{3}\left(\delta_{\mathrm{H}} 3.74, \mathrm{~s}\right)$ to $\mathrm{H}-5^{\prime}$ and $\mathrm{H}-2$ and $\mathrm{H}-6\left(\delta_{\mathrm{H}} 7.21\right.$, overlap); from $2^{\prime}-\mathrm{OH}\left(\delta_{\mathrm{H}} 5.29\right.$, s) to $3^{\prime}-\mathrm{OCH}_{3}$ ] were observed.

The ${ }^{1} \mathrm{H}$ - and ${ }^{13} \mathrm{C}-\mathrm{NMR}$ spectra of $\mathbf{2}$ [White powder. IR $v_{\max }(\mathrm{KBr}) \mathrm{cm}^{-1}: 3485,2930,1604,1564$. UV $\lambda_{\max }^{\mathrm{MeOH}} \mathrm{nm}$ $(\varepsilon): 204$ (43200), 275 (17500). ESI TOF-MS: $m / z 413$
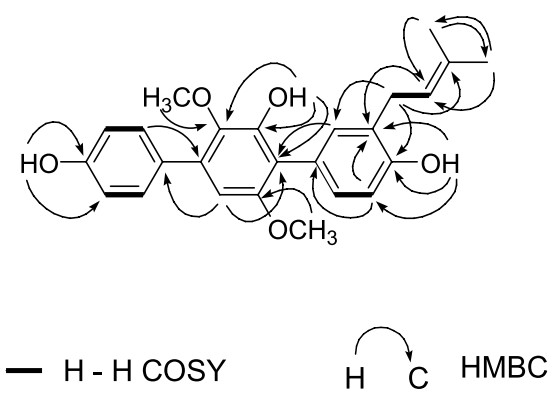

Fig. 4 COSY and key $\mathrm{HMBC}$ correlations of $\mathbf{1}$.
$(\mathrm{M}+\mathrm{Na})^{+}$. HR-ESI TOF-MS: found $\mathrm{m} / \mathrm{z} 413.1756$ $(\mathrm{M}+\mathrm{Na})^{+}$. Calcd for $\mathrm{C}_{25} \mathrm{H}_{26} \mathrm{O}_{4} \mathrm{Na}$ : 413.1729.] (Tables 1 and 2) were closely similar to those of $\mathbf{1}$, except for the signals assignable to the $\mathrm{C}$ ring. The structure of the $\mathrm{C}$ ring was deduced as phenyl group by 2D NMR analysis. Then, $\mathbf{2}$ was defined to be 4 "-dehydoxyl analog of $\mathbf{1} .4$ was identified with 4 "-deoxyterprenin [4], which has been isolated from the culture of a fungus $A$. candidus by Kamigauchi et al.

The molecular formula of 3 [White powder. IR $v_{\max }$ $(\mathrm{KBr}) \mathrm{cm}^{-1}: 3493,2935,1585,1518$. UV $\lambda_{\max }^{\mathrm{MeOH}} \mathrm{nm}(\varepsilon)$ : 203 (45000), 277 (15400). ESI TOF-MS: $m / z 429$ $(\mathrm{M}+\mathrm{Na})^{+}$. HR-ESI TOF-MS: found $\mathrm{m} / \mathrm{z} 429.1683$ $(\mathrm{M}+\mathrm{Na})^{+}$. Calcd for $\mathrm{C}_{25} \mathrm{H}_{26} \mathrm{O}_{5} \mathrm{Na}$ : 429.1678.] was determined as $\mathrm{C}_{25} \mathrm{H}_{26} \mathrm{O}_{5}$ on the basis of NMR and HR-ESI

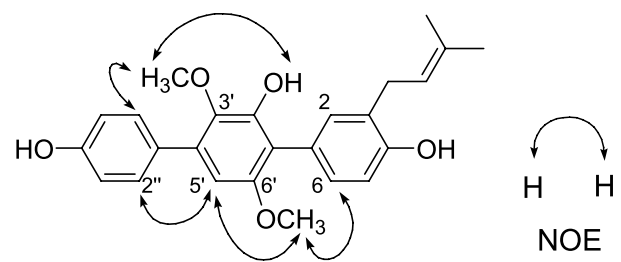

Fig. 5 Key NOE correlations of $\mathbf{1}$

Table $1 \quad{ }^{1} H$-NMR data for prenylterphenyllin (1), 4"-deoxyprenylterphenyllin (2), 4"-deoxyisoterprenin (3), and 4"-deoxyterprenin (4) $\left(600 \mathrm{MHz}\right.$ in $\mathrm{CDCl}_{3}, \delta$ (mult., $\left.\left.J(\mathrm{~Hz})\right)\right)$

\begin{tabular}{|c|c|c|c|c|}
\hline \multirow{2}{*}{ Position } & \multicolumn{4}{|c|}{$\delta(\mathrm{ppm}, J$ in $\mathrm{Hz})$} \\
\hline & 1 & 2 & 3 & 4 \\
\hline 2 & $7.21(\mathrm{~m})$ & 7.23 (s) & 6.99 (s) & 7.06 (s) \\
\hline 5 & $6.88(d, 8.9)$ & $6.88(d, 8.8)$ & $7.00(d, 7.1)$ & $6.96(\mathrm{~s})$ \\
\hline 6 & $7.21(\mathrm{~m})$ & $7.22(d, 8.8)$ & $6.97(d, 7.1)$ & 6.96 (s) \\
\hline $5^{\prime}$ & $6.45(\mathrm{~s})$ & $6.48(\mathrm{~s})$ & $6.47(\mathrm{~s})$ & $6.48(\mathrm{~s})$ \\
\hline $2^{\prime \prime}$ & $7.52(d, 8.2)$ & $7.63(d, 7.4)$ & $7.62(d, 7.4)$ & $7.64(d, 7.4)$ \\
\hline $3^{\prime \prime}$ & $6.91(d, 8.2)$ & $7.45(t, 7.4)$ & $7.44(\mathrm{dd}, 7.4,7.6)$ & $7.45(t, 7.4)$ \\
\hline $4^{\prime \prime}$ & & $7.37(t, 7.4)$ & $7.38(t, 7.6)$ & $7.37(t, 7.4)$ \\
\hline $5^{\prime \prime}$ & $6.91(d, 8.2)$ & $7.45(t, 7.4)$ & 7.44 (dd, 7.4, 7.6) & $7.45(t, 7.4)$ \\
\hline $6^{\prime \prime}$ & $7.52(d, 8.2)$ & $7.63(d, 7.4)$ & $7.62(d, 7.4)$ & $7.64(d, 7.4)$ \\
\hline $1^{\prime \prime \prime}$ & $3.39(d, 7.1)$ & $3.40(d, 7.2)$ & $4.57(d, 6.8)$ & $4.61(d, 6.7)$ \\
\hline $2^{\prime \prime \prime}$ & $5.39(\mathrm{~m})$ & $5.40(\mathrm{~m})$ & $5.50(\mathrm{~m})$ & $5.24(\mathrm{~m})$ \\
\hline $4^{\prime \prime \prime}$ & 1.78 (s) & 1.79 (s) & $1.71(\mathrm{~s})$ & 1.75 (s) \\
\hline $5^{\prime \prime \prime}$ & 1.76 (s) & 1.77 (s) & 1.79 (s) & 1.81 (s) \\
\hline $3-\mathrm{OH}$ & & & & $5.70(\mathrm{~s})$ \\
\hline $4-\mathrm{OH}$ & 5.29 (s) & 5.19 (s) & 5.71 (s) & \\
\hline $2^{\prime}-\mathrm{OH}$ & $5.90(s)$ & $5.90(s)$ & 5.89 (s) & 5.92 (s) \\
\hline $3^{\prime}-\mathrm{OCH}_{3}$ & 3.45 (s) & 3.44 (s) & 3.44 (s) & 3.44 (s) \\
\hline $6^{\prime}-\mathrm{OCH}_{3}$ & 3.74 (s) & 3.74 (s) & 3.74 (s) & 3.75 (s) \\
\hline 4"-OH & 5.32 (s) & & & \\
\hline
\end{tabular}


Table $2{ }^{13} \mathrm{C}-\mathrm{NMR}$ data for $\mathbf{1}, \mathbf{2}, \mathbf{3}$, and $\mathbf{4}\left(150 \mathrm{MHz}\right.$ in $\left.\mathrm{CDCl}_{3}\right)$

\begin{tabular}{|c|c|c|c|c|}
\hline \multirow{2}{*}{ Position } & \multicolumn{4}{|c|}{$\delta_{\mathrm{C}}(\mathrm{ppm})$} \\
\hline & 1 & 2 & 3 & 4 \\
\hline 1 & 125.1 & 125.1 & 124.5 & 125.9 \\
\hline 2 & 132.3 & 132.3 & 114.6 & 117.0 \\
\hline 3 & 126.5 & 126.4 & 145.5 & 145.5 \\
\hline 4 & 153.6 & 153.7 & 145.2 & 145.2 \\
\hline 5 & 115.6 & 115.6 & 114.2 & 111.5 \\
\hline 6 & 129.8 & 129.9 & 123.7 & 122.2 \\
\hline $1^{\prime}$ & 116.4 & 116.8 & 116.9 & 116.6 \\
\hline $2^{\prime}$ & 147.2 & 147.2 & 147.3 & 147.2 \\
\hline $3^{\prime}$ & 138.7 & 138.8 & 138.8 & 138.8 \\
\hline $4^{\prime}$ & 132.3 & 132.7 & 132.8 & 132.8 \\
\hline $5^{\prime}$ & 103.7 & 103.9 & 104.0 & 103.8 \\
\hline $6^{\prime}$ & 153.4 & 153.5 & 153.5 & 153.4 \\
\hline $1^{\prime \prime}$ & 130.4 & 138.1 & 138.1 & 138.1 \\
\hline $2^{\prime \prime}$ & 130.0 & 128.8 & 128.8 & 128.7 \\
\hline $3^{\prime \prime}$ & 115.3 & 128.5 & 128.5 & 128.4 \\
\hline $4^{\prime \prime}$ & 155.3 & 127.5 & 127.5 & 127.4 \\
\hline $5^{\prime \prime}$ & 115.3 & 128.5 & 128.5 & 128.4 \\
\hline $6^{\prime \prime}$ & 120.0 & 128.8 & 128.8 & 128.8 \\
\hline $1^{\prime \prime \prime}$ & 29.8 & 30.0 & 65.7 & 65.6 \\
\hline $2^{\prime \prime \prime}$ & 121.8 & 121.8 & 119.3 & 119.4 \\
\hline $3^{\prime \prime \prime}$ & 134.6 & 134.7 & 138.7 & 138.7 \\
\hline $4^{\prime \prime \prime}$ & 17.9 & 17.9 & 18.2 & 17.9 \\
\hline $5^{\prime \prime \prime}$ & 25.8 & 25.8 & 25.8 & 25.8 \\
\hline $3^{\prime}-\mathrm{OCH}_{3}$ & 60.5 & 60.7 & 60.9 & 60.7 \\
\hline $6^{\prime}-\mathrm{OCH}_{3}$ & 56.0 & 56.0 & 56.0 & 56.0 \\
\hline
\end{tabular}
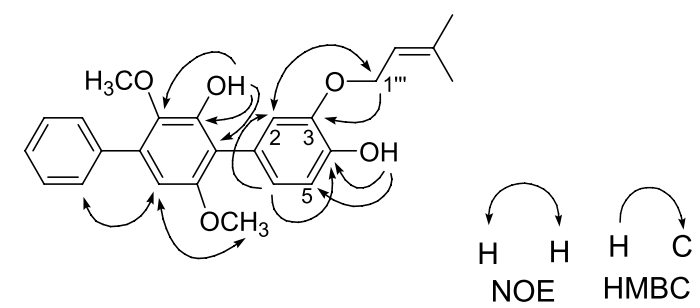

Fig. 6 Key NOE and HMBC correlations of 3.

TOF-MS analysis. The ${ }^{1} \mathrm{H}$ - and ${ }^{13} \mathrm{C}-\mathrm{NMR}$ spectra of $\mathbf{3}$ were almost superimposable to those of $\mathbf{4}$ (Tables 1 and 2), and 3 was presumed to be an isomer of $\mathbf{4}$ concerning with position of the $O$-isoprenyl group. As shown in Fig. 6, the position of the $O$-isoprenyl group in $\mathbf{3}$ was defined based on the HMBC correlations [from $\mathrm{H}-1^{\prime \prime \prime}\left(\delta_{\mathrm{H}} 4.57,2 \mathrm{H}\right.$, d, $J=6.8 \mathrm{~Hz})$ to $\mathrm{C}-3\left(\delta_{\mathrm{C}} 145.5\right)$; from $4-\mathrm{OH}\left(\delta_{\mathrm{H}} 5.71, \mathrm{~s}\right)$ to C$4\left(\delta_{\mathrm{C}} 145.2\right)$ and $\mathrm{C}-5\left(\delta_{\mathrm{C}} 114.2\right)$; from H-6 $\left(\delta_{\mathrm{H}} 6.97, \mathrm{~d}\right.$,
$J=7.1 \mathrm{~Hz})$ to $\mathrm{C}-2\left(\delta_{\mathrm{C}} 114.6\right)$ and $\left.\mathrm{C}-4\right]$ and the NOE correlation between $\mathrm{H}-1^{\prime \prime \prime}$ and $\mathrm{H}-2\left(\delta_{\mathrm{H}} 6.99, \mathrm{~s}\right)$.

$\mathbf{1}, \mathbf{2}, \mathbf{3}$, and $\mathbf{4}$ exhibited cytotoxic activity against KB3-1 with $\mathrm{IC}_{50}$ of $8.5,3.0,2.5$, and $4.5 \mu \mathrm{g} / \mathrm{ml}$, respectively. So far, many related compounds have been reported as anti-proliferative agents [5 7]. Recently, Kamigauchi et al. [4] reported that terprenins including 4 exhibited immunosuppressive activity. Further biological study for these terphenyllins and terprenins are under way.

Acknowledgments This work was supported by the Takeda Science Foundation and the Ministry of Education, Culture, Sports, Science, and Technology of Japan.

\section{References}

1. Blunt JW, Copp BR, Hu WP, Munro MHG, Northcote PT, Prinsep MR. Marine natural products. Nat Prod Rep 24: 
31-86 (2007)

2. Pietra F. Secondary metabolites from marine microorganisms: bacteria, protozoa, algae and fungi. Achievements and prospects. Nat Prod Rep 14: 453-464 (1997)

3. Klich MA. Identification of common Aspergillus species. Centraalbureau voor Schimmelcultures, Utrecht, The Netherlands (2002)

4. Kamigauchi T, Sakazaki R, Nagashima K, Kawamura Y, Yasuda Y, Matsushima K, Tani H, Takahashi Y, Ishii K, Suzuki R, Koizumi K, Nakai H, Ikenishi Y, Terui Y. Terprenins, novel immunosupressants produced by Aspergillus candidus. J Antibiot 51: 445-450 (1998)
5. Takahashi C, Yoshihira K, Natori S, Umeda M. The structures of toxic metabolites of Aspergillus candidus. I. The compounds A and E, cytotoxic $p$-terphenyls. Chem Pharm Bull 24: 613-620 (1976)

6. Kurobane I, Vining LC, McInnes AG, Smith DG. 3Hydroxyterphenyllin, a new metabolite of Aspergillus candidus. Structure elucidation by ${ }^{1} \mathrm{H}$ and ${ }^{13} \mathrm{C}$ nuclear magnetic resonance spectroscopy. J Antibiot 32: 559-564 (1979)

7. Kobayashi A, Takemoto A, Koshimizu K, Kawazu K. pTerphenyls with cytotoxic activity toward sea urchin embryos. Agric Biol Chem 49: 867-868 (1985) 